Rev. Biol. Trop. 52(1): 249-259, 2004

www.ucr.ac.cr www.ots.ac.cr www.ots.duke.edu

\title{
Mamíferos de la Reserva de la Biosfera “La Sepultura”, Chiapas, México
}

\author{
Eduardo Espinoza Medinilla ${ }^{1}$, Epigmenio Cruz $^{2}$, Iván Lira ${ }^{3}$ \& Ignacio Sánchez ${ }^{1}$ \\ 1 El Colegio de la Frontera Sur. Carr. Panamericana y Periférico Sur, Barrio de Ma Auxiliadora; \\ emedinilla@sclc.ecosur.mx \\ 2 Instituto de Historia Natural y Ecología. ZooMAT. Calz. Cerro Hueco S/N Col. El Zapotal. Apdo. Postal 6 Tuxtla \\ Gutiérrez, Chiapas, México C.P. 29000; cruz5910@prodigy.net.mx \\ 3 Universidad del Mar - Campus Puerto Escondido. Puerto Escondido, San Pedro Mixtepec, Juquila, CP 71980, Oaxaca, \\ México; ilira@zicatela.umar.mx
}

Recibido 18-VIII-2000. Corregido 11-X-2001. Aceptado 08-I-2002.

\begin{abstract}
A species list was elaborated for La Sepultura Biosphere Reserve, Chiapas, Mexico. Line transects and direct observations were made, national and international databases were consulted, and animal traces were registered. Animals were captured during the period May 1994 - December 1999. A total of 848 specimen (248 furs and skulls), 32 tracks in plaster, and 9 osseous fragments were collected. A total of 98 species, 70 genera, 29 families, and 10 orders compose the species list. Of these species $87.7 \%$ belong to the orders Chiroptera, Rodentia and Carnivora and 21 species were classified according to some kind of legal protection. These data represent a solid and actualized database useful for designing management and conservation plans for this Biosphere Reserve.
\end{abstract}

Key words: Chiapas, mammals, México, La Sepultura Biosphere Reserve.

La Reserva de la Biosfera La Sepultura, al noroeste de Chiapas, México, se extiende sobre una superficie de 192734 ha. La variedad altitudinal, topográfica, climática y edáfica de la región ha producido una gran cantidad de ecosistemas, encontrándose en este sitio 8 de los 18 tipos de vegetación primaria del estado de Chiapas: bosque lluvioso de montaña, bosque de niebla, chaparral de niebla, bosque de pino-encino, bosque de pino, selva mediana subperennifolia y subcaducifolia, selva baja caducifolia; así como la presencia de potreros, cultivos de maíz, frijol y caficultura orgánica (Breedlove 1981). En los estudios iniciales sobre el área, para su establecimiento como reserva, se habían registrado 49 especies de mamíferos, 143 especies de aves, 48 especies de reptiles y numerosas especies de anfibios, peces e invertebrados no determinadas (Hernández 1994).
Los listados de flora y fauna son ampliamente usados para catalogar observaciones de plantas y animales en el campo. Estos proveen de información sólida y confiable sobre cambios en las poblaciones, su fenología y patrones de abundancia geográfica a escala local, regional y global (Droege et al. 1998, Timmock y Vaughan 2002, Navarro Fernández et al. 2003). Por lo anterior, se consideró, que con base en la información de colecciones nacionales y extranjeras, publicaciones y trabajo de campo, se elaborara un listado, que permita en un futuro, proponer medidas y criterios de conservación congruentes hacia la fauna de mamíferos que esta reserva posee.

\section{MATERIALES Y MÉTODOS}

La Reserva de la Biosfera "La Sepultura" (REBISE), con una extensión de 192734 ha, 
es decretada el 5 de junio de 1995. Se ubica en el Estado de Chiapas entre los $16^{\circ} 00^{\prime}$ y $16^{\circ} 29^{\prime}$ $\mathrm{N}$ y $93^{\circ} 24^{\prime}$ y $94^{\circ} 07^{\prime} \mathrm{O}$, dentro de la región fisiográfica de la Sierra Madre de Chiapas, región sumamente accidentada, que se eleva desde los 25 hasta los 2550 msnm, altura máxima alcanzada por el Cerro Tres Picos (Anónimo 1995).

Para esta región, se distinguen dos zonas: una húmeda, caracterizada por una alta precipitación pluvial y la cual abarca la porción Este de la comarca El Soconusco, con selvas medianas y bosque mesófilo de montaña. Y una segunda región, que se caracteriza por ser menos húmeda y que comprende una porción del Istmo de Tehuantepec, que según Goodwin (1969) es una de las regiones más secas del Pacífico, por lo que las asociaciones vegetales características son las selvas bajas caducifolias y los matorrales xerófitos (Breedlove 1981, Miranda 1975). En su conjunto, la reserva es considerada como una zona de alta diversidad biológica y elevado endemismo (Hernández 1994).

Durante los últimos doce años personal del Instituto de Historia Natural y Ecología (IHNE) ha realizado visitas periódicas con el fin de obtener un listado con la mayor información posible sobre la fauna del lugar (Anónimo 1991). Pero fue a partir de mayo de 1994 que se inició una serie de visitas sistemáticas con el establecimiento de 20 transectos lineales, que presentaban longitudes entre los 0.7 a $12 \mathrm{~km}$ de longitud máxima, y de 1 a 5 m de ancho en siete tipos de hábitat.

Los transectos se recorrieron cuando menos una vez por mes, registrando todas las series de huellas, excretas, restos óseos, echaderos, así como observaciones directas tanto diurnas como nocturnas, procurando evitar sitios con suelo demasiado compacto o pedregoso que impidiera la impresión o recolecta de rastros como señala Aranda (1980).

En el caso de mamíferos de talla pequeña (musarañas, murciélagos y ratones), se colocaron en los transectos, 100 trampas Sherman y 20 Tomahawk, por cada viaje. Durante las noches, entre las 18:00 y las 01:00 horas, se ins- talaron redes de niebla con $11 \mathrm{~m}$ de longitud, éstas variaron en número de acuerdo a la localidad, no siendo menor de tres por noche a un máximo de seis.

Los ejemplares fueron preparados de acuerdo a la técnica de Ramírez Pulido et al. (1989). La determinación taxonómica se llevó a cabo de acuerdo a Hall (1981), Medellín et al. (1997) y Villa (1966). En algunas determinaciones fue necesaria la comparación con ejemplares de la Colección Nacional de Mamíferos del Instituto de Biología de la Universidad Nacional Autónoma de México. Todos los ejemplares fueron depositados en la Colección Zoológica Regional del Sureste de México "Miguel Álvarez del Toro" del IHNE.

Fueron consultadas las bases de datos, en 26 museos nacionales y extranjeros, referentes a todos los especímenes que se encuentran en su acervo para el Estado de Chiapas, de éstos, se observaron las localidades específicas dentro del área de la reserva. Una vez verificada esta información, se integraron algunas especies a nuestro listado. Con base en éste se realizaron tres tipos de análisis, el primero sobre la afinidad biogeográfica de cada una de las especies, el segundo por su tipo de alimentación y el tercero por el estado de conservación de acuerdo a Arita (1993), Arita y Ceballos (1997), Anónimo (2001) y Anónimo (2002).

\section{RESULTADOS}

Se capturaron 848 ejemplares de mamíferos, de los cuales 590 (69.5\%) fueron liberados y 258 preparados como ejemplares de museo. También se recolectaron 32 huellas en moldes de yeso y nueve restos óseos. Durante los recorridos tanto diurnos como nocturnos se lograron observaciones directas de las especies. De la consulta a bases de datos de los 26 museos mexicanos y extranjeros, únicamente 11 presentaron colectas de esta zona, por lo que se obtuvieron 456 registros para la reserva, de los cuales la mayor parte corresponden al periodo de 1950 a 1970. 


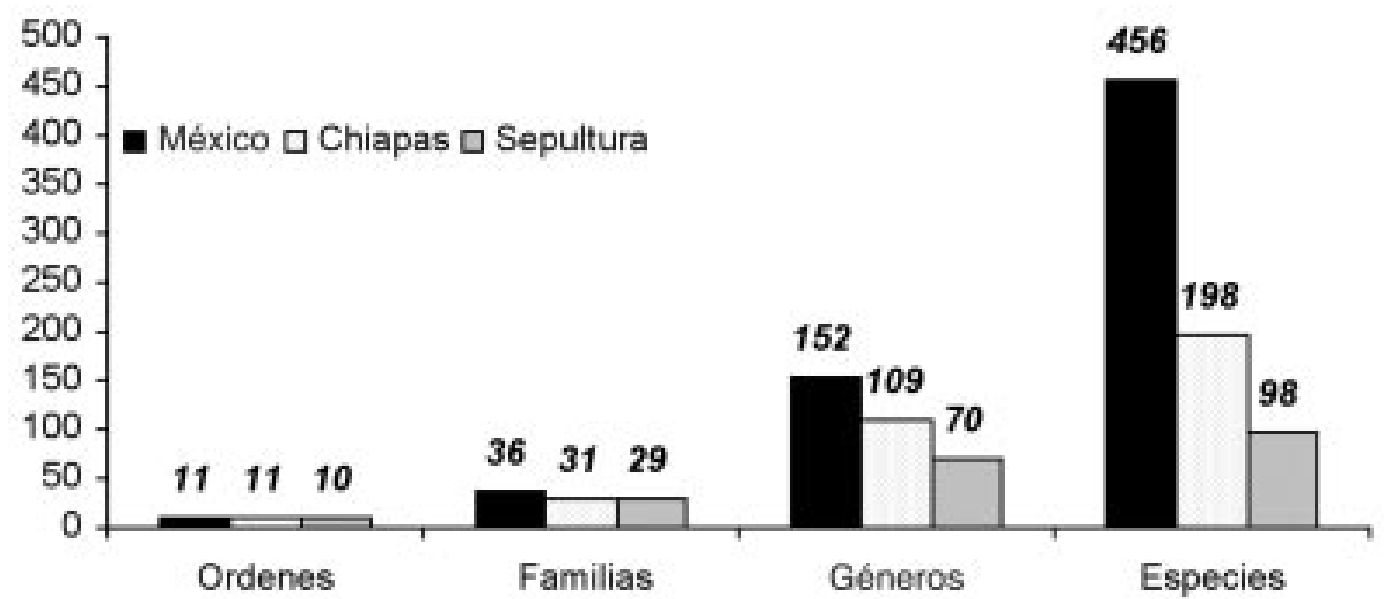

Fig. 1. Comparación de la riqueza mastofaunística de México, Estado de Chiapas y REBISE.

Se obtuvo un total de 98 especies de mamíferos (Anexo 1), 18 de éstas son ejemplares colectados en la zona por otras instituciones, por lo que fue necesario verificar a las especies. Se presenta el primer registro para el Estado de Chiapas de Marmosa canescens (Ratón tlacuache). Las especies se encuentran agrupadas en 10 órdenes, 29 familias y 70 géneros. Los órdenes con mayor representación de especies fueron Chiroptera, Rodentia y Carnivora con un $86.7 \%$ del total de las especies. Considerando que México tiene 452 especies de mamíferos terrestres (Arita y Ceballos 1997), la REBISE representa un $21.6 \%$ del total de especies. En el caso del Estado de Chiapas, representa el $49.4 \%$ del total de especies (Fig. 1).

El orden con mayor número de especies es Chiroptera, de las cuales el $91.02 \%$ pertenecen a las familias Phyllostomidae, Vespertilionidae, Emballonuridae y Mormoopidae con $60 \%, 17.7 \%, 6.6 \%$ y $6.6 \%$ respectivamente.

Para el orden Rodentia, el segundo en mayor representatividad, el $73.8 \%$ de las especies pertenecen a las familias Sciuridae, Heteromyidae y Muridae, con el $13.04 \%, 13.04 \%$ y $47.8 \%$ respectivamente. En el caso de los carnívoros las familias con mayor representatividad fueron Procyonidae (23.5\%), Mustelidae $(35.2 \%)$ y Felidae (29.4\%).
Con respecto a su origen, la mastofauna de la reserva es principalmente Neotropical (NT) (50\% de las especies). Las especies compartidas entre Norte y Sudamérica (NS) son el 29\%, las Mesoamericanas (MA) 13\%, las endémicas a México (MX) constituyen un $4 \%$ y las Neárticas (EA) $4 \%$ (Fig. 2).

De acuerdo a su forma de alimentación, los mamíferos de la REBISE se pueden agrupar en 14 categorías diferentes. Un porcentaje

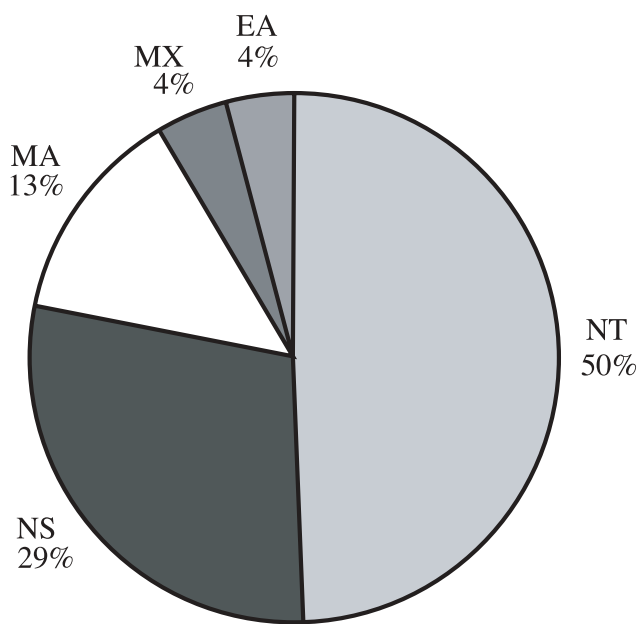

Fig. 2. Origen de las especies de acuerdo a su afinidad biogeográfica. NT=Neotropical; NS= Compartidas entre Norte y Sudamérica; $\mathrm{MA}=$ Mesoamericanas; MX=Endémicas de México; EA=Neárticas. 


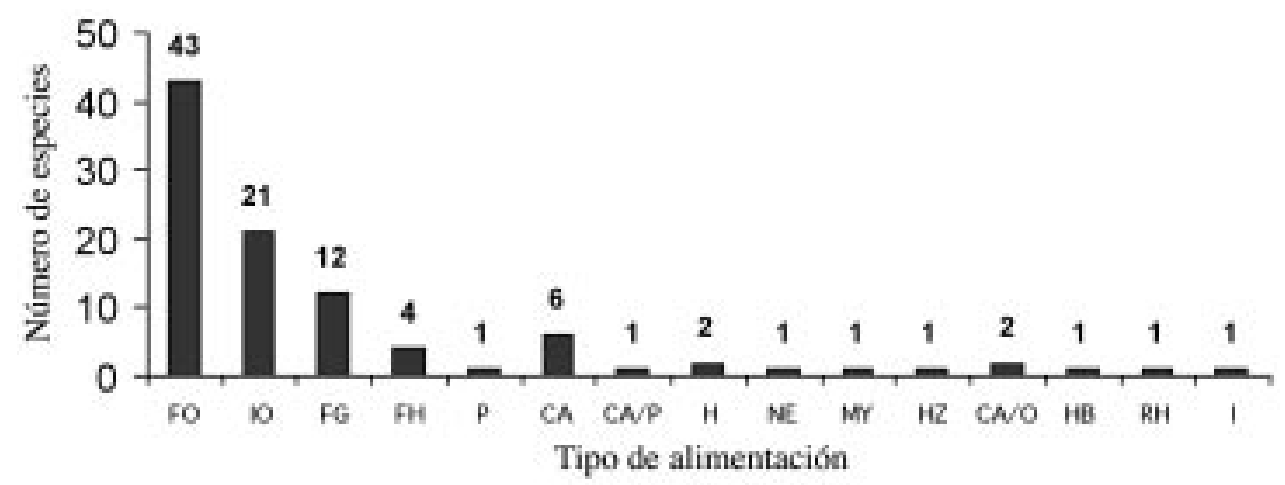

Fig. 3. Tipo de alimentación de los mamíferos en la REBISE.

elevado de especies, el $44.32 \%$, son frugívoroomnívoras (FO), las insectívoro-omnívoras están representadas por un $21.64 \%$ (IO), las frugívoro-granívoras por un $12.37 \%$ (FG), las especies carnívoras constituyen un $6.18 \%$ (CA), las hematófagas un $2.06 \%(\mathrm{H})$, las frugívoro-herbívoras un $4.12 \%(\mathrm{FH})$, las carnívoroomnívoras un $2.06 \%(\mathrm{CA} / \mathrm{O})$, y las piscívoras (P), carnívoro-piscívoras (CA/P), nectívoras (NE), mirmecófagas (MY), herbívoro-pasteadoras (HZ), herbívoro-ramoneadoras (HB), raizívoras $(\mathrm{RH})$ e insectívoras (I), representan en cada caso el $1.03 \%$ (Fig. 3).

Por otra parte, al menos 20 especies se encuentran clasificadas en alguna categoría de riesgo de extinción. Unas nueve especies de mamíferos de La Sepultura se encuentran en la lista de especies en peligro de la Unión Internacional para la Conservación de la Naturaleza (IUCN). La Convención Internacional sobre Tráfico de Especies en Peligro de Flora y Fauna (CITES) considera a 15 especies dentro de alguno de sus tres apéndices, muy similar a lo propuesto por la Norma Oficial Mexicana (Anónimo 2001), en la cual sólo 15 especies presentan algún criterio de conservación. Parece no existir concordancia entre los criterios empleados por cada una de estas instancias, pues solamente ocho especies son consideradas por al menos dos de ellas (Fig. 4).

Aproximadamente 23 especies de mamíferos son aprovechadas de alguna manera por los pobladores de la región; 14 especies son utilizadas casi exclusivamente para consumo local, ya sea utilizándolas como alimento o bien como mascotas; entre las más comunes se encuentran: Didelphis marsupialis, D. virginiana, Tapirus bairdii, Agouti paca, Dasyprocta mexicana, Sylvilagus floridanus, Ateles geoffroyi, Odocoileus virginianus, Mazama americana, Pecari tajacu y Nasua narica. Con fines ornamentales o como mascotas se utilizan: Herpailurus yagouaroundi, Leopardus pardalis, Leopardus wiedii, A. geoffroyi, y Procyon lotor o con ambos propósitos: Potos flavus, Sciurus aureogaster, N. narica y P. lotor (Cruz 2000).

Dasypus novemcinctus y A. geoffroyi son las especies a las que se les da un número mayor de usos, pues además de ser utilizados como alimento, adornos o mascotas por la gente

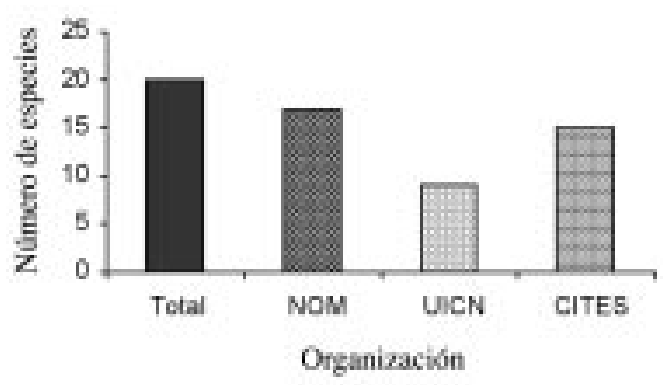

Fig. 4. Estado de conservación de los mamíferos en la REBISE. 
local, son comercializados por creer que tienen propiedades medicinales (Cruz 2000).

\section{DISCUSIÓN}

La mastofauna de la Reserva de la Biosfera La Sepultura se encuentra representada por un total de 98 especies, con este estudio se han aportado 37 nuevos registros a los 61 que se tenían para el área, 18 de ellos fueron obtenidos de colecciones. Es importante señalar que $M$. canescens es un nuevo registro, por lo que aumenta el rango de distribución hasta el Estado de Chiapas, probablemente siguiendo la distribución de la selva baja caducifolia.

De acuerdo a listas de mamíferos que se tienen de otras áreas naturales protegidas en el estado, se puede observar que la REBISE ocupa el tercer lugar en cuanto a número de especies registradas, seguida por la Selva el Ocote con 97 especies (Navarrete et al. 1996), el Cañón del Sumidero con 46 especies (Gálvez 1990), siendo El Triunfo con 112 especies (Espinoza et al. 1998) y la Selva Lacandona con 124 especies (March y Aranda 1992) las primeras reservas que albergan la mayor diversidad de especies.

Entre los principales problemas de la región se encuentran: la expansión de la frontera agropecuaria, los incendios forestales y la creación de nuevos centros de población. Dentro del área, además de las actividades antes mencionadas, se realiza un aprovechamiento no regulado de recursos maderables (pino $\mathrm{Pi}$ nus sp., encino Quercus sp., cedro Cedrella sp., amate Ficus sp.) y no maderables (Palma camedora o Chamaedorea sp.) (Anónimo 1999).

Frecuentemente se practica la cacería de autoconsumo, la comercial y el tráfico de animales. La primera constituye una alternativa de complemento a la dieta familiar y las dos últimas se llevan a cabo como alternativas del ingreso económico. La cacería de autoconsumo es llevada a cabo prácticamente por todas las comunidades asentadas dentro de la Sierra; es una práctica comúnmente asociada a la reco- lección de la palma Chamaedora sp., aunque oficialmente, la región presenta veda permanente al aprovechamiento de todas las especies de flora y fauna (Anónimo 1999).

La fauna de mamíferos de la REBISE debido a la influencia de las especies compartidas entre Norte y Sudamérica, aunadas a las especies neárticas, ofrece un panorama único en su conjunto de especies. La riqueza de especies es superior a la presentada por la Reserva de la Biosfera Selva el Ocote y al Parque Nacional Cañón del Sumidero, considerando que esta reserva es de las más afectadas y amenazadas con respecto a las actividades antropocéntricas que ahí se desarrollan.

En vista de que una alta proporción de especies enfrenta algún tipo de amenaza de extinción, una estrategia de conservación sería el establecimiento de Unidades de Manejo y Aprovechamiento (UMAS), plan desarrollado por el gobierno mexicano, además la implementación de los sistemas agrosilvopastoriles y agroforestales, que al parecer son de los más benéficos para la conservación de la fauna silvestre; debido a esto es importante conducir investigaciones dirigidas a la integración de dichas acciones dentro de la reserva.

Cabe destacar que la lista de mamíferos que se presenta es parcial, falta seguir realizando más estudios y observaciones en el lugar. Principalmente en los grupos de roedores, quirópteros e insectívoros. Es necesario resaltar la importancia de la reserva como refugio de comunidades de mamíferos, principalmente de aquellas de distribución restringida y que al parecer, no habitan en otras áreas naturales protegidas de México y Chiapas.

\section{AGRADECIMIENTOS}

Al personal del IHNE que colaboró en la realización y observaciones en la reserva, principalmente a Antonio Ramírez Velásquez, Fredi M. Herrera Pérez y de manera muy especial a la familia Canseco Cruz, por todas las facilidades y apoyo en los viajes de campo. A Alejandro Flamenco y Helda Kramsky por su 
invaluable apoyo en el análisis de las bases de datos. También a todos los revisores anónimos que participaron en la revisión del presente manuscrito, por sus comentarios y observaciones. La mayor parte del trabajo fue financiada por la Comisión Nacional para el Conocimiento y Uso de la Biodiversidad (CONABIO) a través del proyecto P060 y el Proyecto Ecología, Biología y Conservación del Tapir (Tapirus bairdii) en el Estado de Chiapas, perteneciente a la línea de investigación del Instituto de Historia Natural y Ecología.

\section{RESUMEN}

Con el objeto de conocer la riqueza de mamíferos de la Reserva de la Biosfera La Sepultura, fue elaborado un listado de especies para esta área natural protegida. A través de transectos lineales, donde se realizaron observaciones directas, búsqueda de rastros y captura de ejemplares, durante el periodo de mayo de 1994 a diciembre de 1999, además de la revisión de bases de datos nacionales y extranjeras; con registros de esta localidad, se pudo recolectar 848 especimenes ( 248 pieles y cráneos), 32 huellas en molde de yeso y nueve fragmentos óseos. Se obtuvo un listado compuesto de 98 especies, 70 géneros, 29 familias y 10 órdenes. El $86.7 \%$ de estas especies pertenecen a los órdenes Chiroptera, Rodentia y Carnivora. Por lo menos 21 especies están clasificadas dentro de alguna categoría de conservación. Los datos obtenidos de este manuscrito son una base sólida y actualizada y pueden ser usados como parte de los planes de manejo y conservación para esta reserva.

\section{REFERENCIAS}

Álvarez del Toro, M. 1991. Los Mamíferos de Chiapas. Gobierno del Estado de Chiapas. DIF-Chiapas, Instituto Chiapaneco de Cultura. Tuxtla Gutiérrez, Chiapas, México. 133 p.

Anónimo. 1991. Propuesta área natural protegida "Zona sujeta a Conservación ecológica La Sepultura". Resumen ejecutivo. Instituto de Historia Natural, Depto. de Áreas Naturales, Tuxtla Gutiérrez, Chiapas, México. 15 p.

Anónimo. 1995. Reservas de la Biosfera y otras Áreas Naturales Protegidas de México. Instituto Nacional de Ecología (INE), Comisión Nacional para el Conocimiento y Uso de la Biodiversidad (CONABIO), Secretaria del Medio Ambiente, Recursos Naturales y Pesca. México D.F., México.
Anónimo. 1999. Programa de Manejo de la Reserva de La Biosfera El Triunfo, México. Instituto Nacional de Ecología (INE), Secretaría del Medio Ambiente Recursos Naturales y Pesca (SEMARNAP), México D.F., México. pp. 107-247.

Anónimo. 2001. Norma Oficial Mexicana NOM-059ECOL-2001. Protección ambiental - Especies nativas de México de flora y fauna silvestres Categorías de riesgo y especificaciones para su inclusión, exclusión o cambio - Lista de especies en riesgo. Secretaría de Medio Ambiente Recursos Naturales y Pesca (SEMARNAT). México.

Anónimo. 2002. 2000 IUCN Red list of threatened animals. IUCN Species Survival Commission, Gland, Suiza. (Consultado: 12/VII/200O y 24/VIII/2002, http://www.redlist.org).

Aranda, J.M. 1980. Importancia y utilidad de los rastros para el estudio de mamíferos silvestres. Tesis de licenciatura, UNAM, México D.F., México. 120 p.

Arita, H.T. 1993. Riqueza de Especies de la Mastofauna de México, pp. 109-128. In R.A. Medellín y G. Ceballos (eds.). Avances en el Estudio de los Mamíferos de México. Asociación Mexicana de Mastozoología, México.

Arita, H.T. \& G. Ceballos. 1997. Los mamíferos de México: Distribución y estado de conservación. Rev. Mex. Mastozoo. 2: 33-71.

Breedlove, D.E. 1981. Flora of Chiapas. Part I. Introduction to the flora of Chiapas. California Academy of Sciences, San Francisco, California, Estados Unidos.

Cruz, A.E. 2000. Usos tradicionales de la fauna silvestre de Chiapas. BARUM, Rev. Inst. Hist. Nat. 25: 25-28.

Espinoza, M., E.A. Anzures \& E. Cruz. 1998. Los Mamíferos de El Triunfo. Rev. Mex. Mastozoo. 3: 79-94.

Droege, S., A. Cyr \& J. Larivee. 1998. Checklists: An under-used tool for the inventory and monitoring of plants and animals. Conserv. Biol. 12(5): 1134-1138.

Gálvez, J. 1990. Mastofauna del Parque Nacional "Cañón del Sumidero" Chiapas, México. Tesis de licenciatura, Instituto de Ciencias y Artes de Chipas, Tuxtla Gutiérrez, Chiapas. pp. 89.

Goodwin, G.G. 1969. Mammals from the state of Oaxaca, Mexico in the American Museum of Natural History. Bull. Amer. Mus. Natur. Hist. 141: 1.

Hall, E.R. 1981. The Mammals of North America. Vols. I, II. Wiley. Nueva York, Estados Unidos.

Hernández, Y.A. 1994. Propuesta para establecer el área Natural Protegida Reserva de la Biosfera "La Sepultura", en la porción oeste de la Sierra Madre de 
Chiapas. Tesis de licenciatura,Universidad Veracruzana, Xalapa, Veracruz. 127 p.

March, I. \& M. Aranda. 1992. Mamíferos de la Selva Lacandona, pp. 201-220. In M.A. Vázquez \& M.A. Ramos (eds.). Reserva de la Biosfera Montes Azules, Selva Lacandona: Investigación para su Conservación. Publicaciones Especiales Ecosfera 1: 1-436.

Medellín, R.A., H.T. Arita \& O. Sánchez T. 1997. Identificación de los murciélagos de México: Clave de campo. Publicaciones Especiales, Asociación Mexicana de Mastozoología, México D.F., México.

Miranda, F. 1975. La vegetación de Chiapas. $2^{\text {a }}$ Ed. Ediciones del Gobierno del Estado, Tuxtla Gutiérrez, Chiapas, México.

Navarro Fernández, E., C. Pozo De La Tijera \& E. Escobedo Cabrera. 2003. Afinidad ecológica y distribución actual de Primates (Cebidae) en Campeche, México. Rev. Biol. Trop. 51: 591-600.
Navarrete, D.A., M.P. Alba, I.J. March \& E. Espinoza. 1996. Mamíferos de la Selva El Ocote, Chiapas, pp. 179-207. In M.A. Vázquez. \& I. March M. (eds.).Conservación y Desarrollo Sustentable en la Selva El Ocote, Chiapas. El Colegio de la Frontera Sur en colaboración del Centro de Estudios para la Conservación de los Recursos Naturales, A.C. 419 p.

Ramírez-P.J., Lira. I. Salvador Gaona., C. Müdespacher \& A. Castro-Campillo. 1989. Manejo y Mantenimiento de Colecciones Mastozoológicas. Universidad Autónoma Metropolitana, México, D.F., México.

Timmock, J. \& C. Vaughan. 2002. A Census of Mammal Populations in Punta Leona Private Wildlife Refuge, Costa Rica. Rev. Biol. Trop. 50: 1169-1180.

Villa, R.B. 1966. Los Murciélagos de México. Instituto de Biología, UNAM., México, D.F., México.

Wilson, D.E. \& D.M. Reeder (eds). 1993. Mammal species of the world: A Taxonomic and Geographic Reference. $2^{\text {nd }}$ Ed. Smithsonian, Washington D.C., Estados Unidos. 


\section{ANEXO I}

\section{Lista preliminar de las especies de mamíferos de la Reserva de la Biosfera "La Sepultura"}

Los órdenes se mencionan en la secuencia filogenética propuesta por Wilson y Reeder (1993).

Las familias, géneros y especies se listan en orden alfabético. El nombre común de las especies está basado en las publicaciones realizadas por Alvarez del Toro (1991)

Las abreviaturas en las columnas son como sigue:

DIST $=$ distribución $(\mathrm{NT}=$ neotropical; $\mathrm{MA}=$ mesoamericano; $\mathrm{MX}=$ endémico de México; NS = compartidos en Norteamérica y Sudamérica; $\mathrm{EA}=$ neártico).

NOM = conservación según NOM-059ECOL-2000 ( $\mathrm{E}=$ extinta en medio silvestre; $\mathrm{P}$ $=$ peligro de extinción; $\mathrm{A}=$ amenazada; $\mathrm{PR}=$ sujeta a protección especial).

IUCN $=$ categoría según IUCN (EX = extinta; EW = extinta en estado silvestre; $\mathrm{CR}=$ críticamente amenazada; $\mathrm{EN}$ = en peligro; $\mathrm{VU}$ $=$ vulnerable; $\mathrm{LC}: \mathrm{NT}=$ en menor riesgo).

CITES $=$ apéndice según CITES $(\mathrm{I}=$ podrían ser extinguidas por el tráfico; $\mathrm{II}=$ podrían extinguirse si no se controla el tráfico; III $=$ reguladas por algún socio del tratado).

Aliment $=$ clasificación del tipo de dieta (FO = frugívoro-omnívoro; $\mathrm{I}=$ insectívoro; $\mathrm{IO}=$ insectívoro-omnívoro; $\mathrm{FG}=$ frugívoro-granívoro, $\mathrm{FH}$ = frugívoro-herbívoro; $\mathrm{HB}=$ herbívoro-ramoneador; $\mathrm{MY}=$ mirmecófago; HZ = herbívoro-pasteador; $\mathrm{CA}=$ carnívoro; $\mathrm{CA} / \mathrm{O}=$ carnívoro-omnívoro; $\mathrm{CA} / \mathrm{P}=$ carnívoro-piscívoro; $\mathrm{NE}=$ nectívoro; $\mathrm{H}=$ hematófago; $\mathrm{PI}=$ piscívoro; $\mathrm{RH}=$ raizívoro.

Las fuentes de información son las siguientes:

\begin{tabular}{|c|c|c|}
\hline $\mathrm{N}^{\circ}$ & $\begin{array}{l}\text { Acrónimo de } \\
\text { Colecciones }\end{array}$ & Nombre \\
\hline 1 & IHNMASTO & Colección Zoológica Regional del Instituto de Historia Natural. Colección Mastozoológica. \\
\hline 2 & ECO.SC.M. & $\begin{array}{l}\text { Colección Mastozoológica de El Colegio de la Frontera Sur. San Cristóbal de las Casas. } \\
\text { Chiapas. }\end{array}$ \\
\hline 3 & IBUNAM & $\begin{array}{l}\text { Colección Mastozoológica del Instituto de Biología de la Universidad Nacional Autónoma } \\
\text { de México. }\end{array}$ \\
\hline 4 & & Base de datos del Atlas Mastozoológico de México. \\
\hline 5 & ENCB & Escuela Nacional de Ciencias Biológicas, Instituto Politécnico Nacional. \\
\hline 6 & IUMNH & Illinois University, Museum of Natural History. \\
\hline 7 & UMMZ & University of Michigan Museum of Zoology Michigan. \\
\hline 8 & FMNH & Field Museum of Natural History, Chicago. \\
\hline 9 & $\mathrm{KU}$ & Kansas University, Museum of Zoology. \\
\hline 10 & $\mathrm{MZFC}$ & $\begin{array}{l}\text { Museo de Zoología "Alfonso L. Herrera" de la Facultad de Ciencias de la Universidad Na- } \\
\text { cional Autónoma de México. }\end{array}$ \\
\hline 11 & Texas A\&M & Texas Comparative Wildlife Collection. Departament of Wildlife Science, Texas A\&M. \\
\hline
\end{tabular}

En el mismo cuadro, $(*)$ denota que esa especie no fue recolectada durante el estudio, pero los ejemplares, se encuentran en la colección y fueron revisados por los autores. 


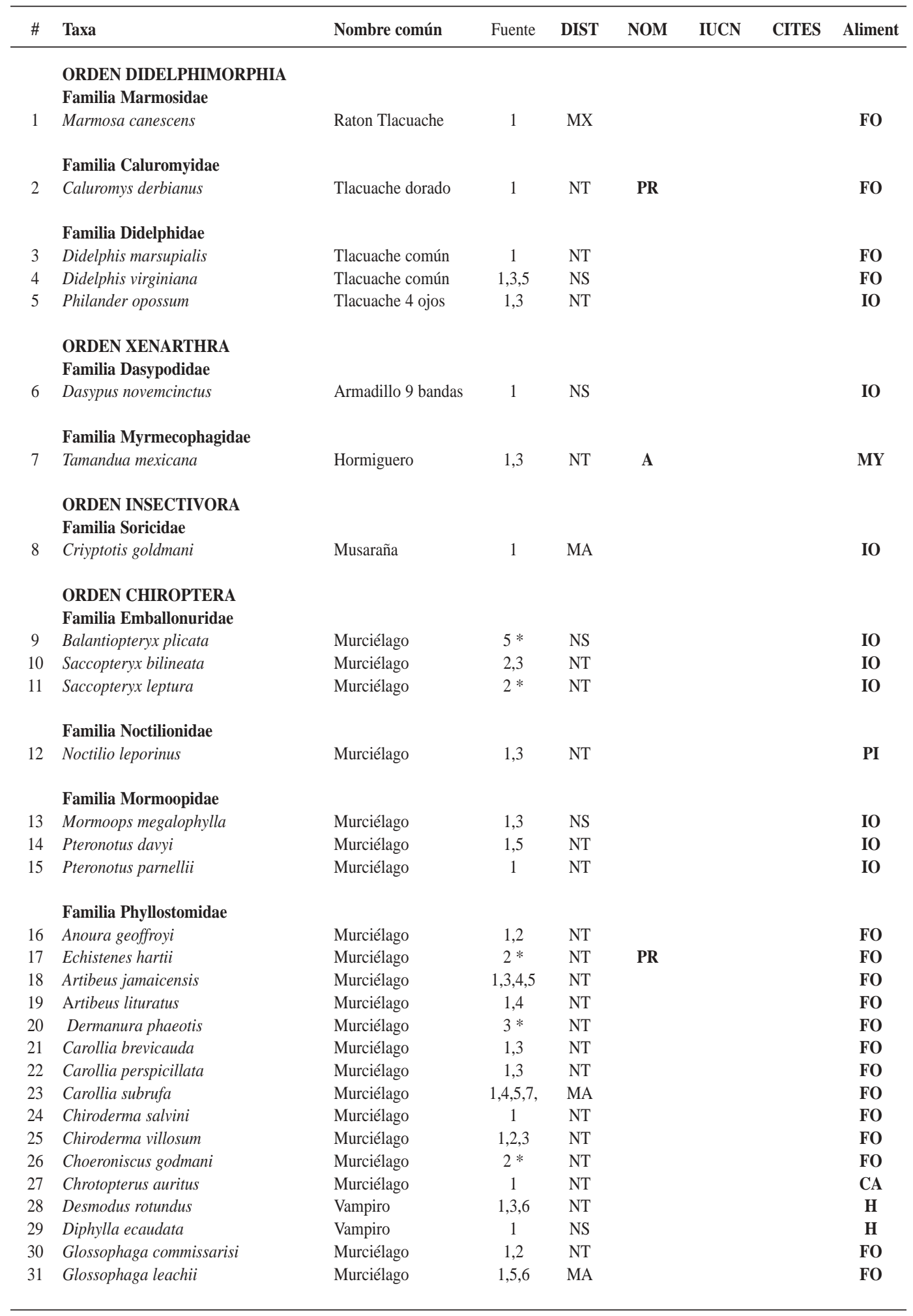




\begin{tabular}{|c|c|c|c|c|c|c|c|c|}
\hline$\#$ & Taxa & Nombre común & Fuente & DIST & NOM & IUCN & CITES & Aliment \\
\hline 32 & Glossophaga morenoi & Murciélago & 1,2 & MX & & & & FO \\
\hline 33 & Glossophaga soricina & Murciélago & $1,3,6,7$ & NT & & & & FO \\
\hline 34 & Hylonycteris underwoodi & Murciélago & 1 & MA & & & & NE \\
\hline 35 & Micronycteris megalotis & Murciélago & 5,7 & NT & & & & FO \\
\hline 36 & Micronycteris sylvestris & Murciélago & 1 & NT & & & & FO \\
\hline 37 & Phyllostomus discolor & Murciélago & $2,3 *$ & NT & & & & FO \\
\hline 38 & Sturnira lilium & Murciélago & 1 & NT & & & & FO \\
\hline 39 & Sturnira ludovici & Murciélago & 1,3 & NT & & & & FO \\
\hline 40 & Uroderma bilobatum & Murciélago & $1,2,3,8$ & NT & & & & FO \\
\hline 41 & Uroderma magnirostrum & Murciélago & $2 *$ & NT & & & & FO \\
\hline \multirow[t]{2}{*}{42} & Vampyressa pusilla & Murciélago & 1 & NT & & & & FO \\
\hline & Familia Vespertilionidae & & & & & & & \\
\hline 43 & Eptesicus brasiliensis & Murciélago & $3 *$ & NT & & VU & & IO \\
\hline 44 & Eptesicus furinalis & Murciélago & $3 *$ & NT & & & & IO \\
\hline 45 & Lasiurus borealis & Murciélago & $3 *$ & EA & & & & IO \\
\hline 46 & Lasiurus ega & Murciélago & $3 *$ & EA & & & & IO \\
\hline 47 & Lasiurus intermedius & Murciélago & $3 *$ & NS & & & & IO \\
\hline 48 & Myotis elegans & Murciélago & 2,3 & MA & & & & IO \\
\hline 49 & Myotis keaysi & Murciélago & 2 & NT & & & & IO \\
\hline \multirow[t]{2}{*}{50} & Rhogeessa tumida & Murciélago & $2,5 *$ & NT & & & & IO \\
\hline & Familia Molossidae & & & & & & & \\
\hline 51 & Molossus ater & Murciélago & $1,3,5$ & NT & & & & IO \\
\hline \multirow[t]{2}{*}{52} & Nyctinomops laticaudatus & Murciélago & $2 *$ & NT & & & & IO \\
\hline & Familia Natalidae & & & & & & & \\
\hline \multirow[t]{3}{*}{53} & Natalus stramineus & Murciélago & $3 *$ & NS & & & & IO \\
\hline & ORDEN PRIMATES & & & & & & & \\
\hline & Familia Cebidae & & & & & & & \\
\hline \multirow[t]{2}{*}{54} & Ateles geoffroyi & Mono araña & 1 & MA & $\mathbf{P}$ & VU & II & FH \\
\hline & $\begin{array}{l}\text { ORDEN LAGOMORPHA } \\
\text { Familia Leporidae }\end{array}$ & & & & & & & \\
\hline \multirow[t]{3}{*}{55} & Sylvilagus floridanus & Conejo & 1,4 & NS & & & & $\mathrm{HZ}$ \\
\hline & ORDEN RODENTIA & & & & & & & \\
\hline & Familia Sciuridae & & & & & & & \\
\hline 56 & Sciurus aureogaster & Ardilla gris & 1,4 & NT & & & & FG \\
\hline 57 & Sciurus deppei & Ardilla de selva & 1 & MA & & & & FG \\
\hline \multirow[t]{2}{*}{58} & Glaucomys volans & Ardilla voladora & 1 & NS & $\mathbf{A}$ & & & FG \\
\hline & Familia Geomyidae & & & & & & & \\
\hline \multirow[t]{2}{*}{59} & Orthogeomys grandis & Tuza & 1,3 & MA & & & & RH \\
\hline & Familia Heteromyidae & & & & & & & \\
\hline 60 & Heteromys desmarestianus & Rata de campo & 1 & NT & & & & FG \\
\hline 61 & Liomys pictus & Rata de campo & $1,4,5,6$ & MA & & & & FG \\
\hline \multirow[t]{2}{*}{62} & Liomys salvini & Rata de campo & $1,3,5$ & MA & & & & FG \\
\hline & Familia Muridae & & & & & & & \\
\hline 63 & Baiomys musculus & Ratón & $1,4,5,6$ & MA & & & & FO \\
\hline 64 & Neotoma mexicana & Ratón & 1 & NS & & & & FO \\
\hline
\end{tabular}




\begin{tabular}{|c|c|c|c|c|c|c|c|c|}
\hline$\#$ & Taxa & Nombre común & Fuente & DIST & NOM & IUCN & CITES & Aliment \\
\hline 65 & Olygoryzomys fulvescens & Ratón & $1,4,6$ & NT & & & & FO \\
\hline 66 & Oryzomys palustris & Ratón & 1,4 & NS & & & & FO \\
\hline 67 & Peromyscus boylii & Ratón & 5 & NS & & & & FO \\
\hline 68 & Peromyscus melanophrys & Ratón & $1,4,5$ & MX & & & & FO \\
\hline 69 & Peromyscus mexicanus & Ratón & 1 & MA & & & & FO \\
\hline 70 & Reithrodontomys fulvescens & Ratón & $4 *$ & EA & & & & FO \\
\hline 71 & Reithrodontomys mexicanus & Ratón & $4 *$ & NT & & & & FO \\
\hline 72 & Sigmodon hispidus & Ratón & $1,3,4$ & NS & & & & FG \\
\hline \multirow[t]{2}{*}{73} & Sigmodon mascotensis & Ratón & $3 *$ & MX & & & & FG \\
\hline & Familia Erethizonthidae & & & & & & & \\
\hline \multirow[t]{2}{*}{74} & Sphiggurus mexicanus & Puerco espín & 1 & MA & $\mathbf{A}$ & & III & FG \\
\hline & Familia Agoutidae & & & & & & & \\
\hline \multirow[t]{2}{*}{75} & Agouti paca & Tepezcuintle & 1 & NT & & & III & FG \\
\hline & Familia Dasyproctidae & & & & & & & \\
\hline 76 & Dasyprocta mexicana & Guaqueque negro & 1 & NT & & & & FG \\
\hline \multirow[t]{2}{*}{77} & Dasyprocta punctata & Guaqueque alazán & 1 & NT & & & III & FG \\
\hline & $\begin{array}{l}\text { ORDEN CARNIVORA } \\
\text { Familia Canidae }\end{array}$ & & & & & & & \\
\hline 78 & Urocyon cinereoargenteus & Zorra Gris & $1,4,5$ & NS & & & & FO \\
\hline \multirow[t]{2}{*}{79} & Canis latrans & Coyote & 1 & EA & & & & $\mathrm{CA} / \mathrm{O}$ \\
\hline & Familia Procyonidae & & & & & & & \\
\hline 80 & Bassariscus sumichrasti & Cacomixtle & 1 & MA & PR & LC:NT & III & FO \\
\hline 81 & Nasua narica & Tejón & 1,8 & NS & & & III & FO \\
\hline 82 & Potos flavus & Mico de noche & 1 & NT & PR & & & FO \\
\hline \multirow[t]{2}{*}{83} & Procyon lotor & Mapache & 1 & NS & & & & FO \\
\hline & Familia Mustelidae & & & & & & & \\
\hline 84 & Conepatus mesoleucus & Zorrillo & 1 & NS & & & I & FO \\
\hline 85 & Eira barbara & Viejo de monte & 1 & NT & $\mathbf{P}$ & EN & III & FO \\
\hline 86 & Mephytis macroura & Zorrillo & 1 & NS & & & & IO \\
\hline 87 & Spilogale putorius & Zorrillo & 1 & NS & & & & IO \\
\hline 88 & Mustela frenata & Comadreja & 1 & NS & & & & $\mathrm{CA} / \mathrm{O}$ \\
\hline \multirow[t]{2}{*}{89} & Lontra longicaudis & Nutria & $1,2,5$ & NT & $\mathbf{A}$ & & II & $\mathbf{C A} / \mathbf{P}$ \\
\hline & Familia Felidae & & & & & & & \\
\hline 90 & Herpailurus yagouaroundi & Leoncillo & 1,2 & NS & $\mathbf{A}$ & EN & I & CA \\
\hline 91 & Leopardus pardalis & Ocelote & 1,2 & NS & $\mathbf{P}$ & EN & I & CA \\
\hline 92 & Leopardus wiedii & Tigrillo & 1 & NS & $\mathbf{P}$ & VU & $\mathbf{I}$ & CA \\
\hline 93 & Panthera onca & Jaguar & 1,2 & NS & $\mathbf{P}$ & VU & I & CA \\
\hline \multirow[t]{2}{*}{94} & Puma concolor & Puma & 1 & NS & & & & CA \\
\hline & $\begin{array}{l}\text { ORDEN PERISSODACTY } \\
\text { Familia Tapiridae }\end{array}$ & & & & & & & \\
\hline \multirow[t]{2}{*}{95} & Tapirus bairdii & Tapir & 1 & NT & $\mathbf{P}$ & VU & I & FH \\
\hline & $\begin{array}{l}\text { ORDEN ARTIODACTYLA } \\
\text { Familia Tayassuidae }\end{array}$ & & & & & & & \\
\hline \multirow[t]{2}{*}{96} & Pecari tajacu & Jabalí de collar & 1 & NS & & & II & FH \\
\hline & Familia Cervidae & & & & & & & \\
\hline 97 & Mazama americana & Venado de montaña & 1 & NT & & & & FH \\
\hline 98 & Odocoileus virginianus & Venado cola blanca & 1,6 & NS & & & & HB \\
\hline
\end{tabular}


